\title{
Chemische Biologie
}

\section{Die Länge spezifischer Ubiquitin- ketten beeinflusst ihre Erkennung}

\author{
JOACHIM LUTZ ${ }^{1}$, EVA HÖLLMÜLLER ${ }^{1,2}$, MARTIN SCHEFFNER ${ }^{2}$, ANDREAS MARX ${ }^{1}$, \\ FLORIAN STENGEL ${ }^{2}$ \\ ${ }^{1}$ FACHBEREICH CHEMIE, KONSTANZ RESEARCH SCHOOL CHEMICAL BIOLOGY, \\ UNIVERSITÄT KONSTANZ \\ 2 FACHBEREICH BIOLOGIE, KONSTANZ RESEARCH SCHOOL CHEMICAL BIOLOGY, \\ UNIVERSITÄT KONSTANZ
}

\section{The attachment of differently linked ubiquitin (Ub) chains of varying length to proteins is a prevalent posttranslational modification in eukaryotic cells. We established an affinity enrichment approach for the identification of linkage- and length-selective ubiquitin binding proteins and could show that in addition to the linkage, the length of a $\mathrm{Ub}$ chain is an important determinant for $\mathrm{Ub}$ chain recognition.}

DOI: $10.1007 / \mathrm{s} 12268-020-1421-3$

(C) Die Autoren 2020

Die kovalente Anheftung von Ubiquitin (Ub) an Substratproteine (Ubiquitylierung) ist eine der komplexesten und vielfältigsten posttranslationalen Modifikationen in Eukaryoten [1]. Viele zelluläre Prozesse wie Proteinabbau, Zellteilung und Signaltransduktion werden durch Ubiquitylierung reguliert. Dementsprechend sind viele verschiedene Krankheiten wie Krebs oder neurodegenerative Erkrankungen eng mit Störungen beim Prozess der Ubiquitylierung verbunden [2].

Die Ubiquitylierung wird durch drei Klassen von Enzymen katalysiert [1]:

- E1: Ub-aktivierende Enzyme,

- E2: Ub-konjugierende Enzyme und

- E3: Ub-Ligasen.

Letztere sind maßgeblich an der Bildung einer Isopeptidbindung zwischen der C-terminalen Carboxylgruppe von Ubiquitin und der $\varepsilon$-Amino-Gruppe eines Lysinrests des Substratproteins beteiligt [3]. Über die Anheftung einzelner Ub-Moleküle (Mono-Ubiquitylierung) hinaus kann Ub selbst ebenfalls als Substrat dienen, was zur Bildung von UbKetten (Polyubiquitylierung) führt. Je nachdem welcher der sieben Lysine (K6, K11, K27, K29, K33, K48, K63) - bzw. der N-terminalen Methioninreste) - für die Verknüpfung benutzt wird, werden unterschiedliche Kettentypen gebildet. Somit können Proteine theoretisch mit einer beinahe unendlichen Vielzahl Nach [11, 12], (c) John Wiley \& Sons, Inc. den. Des Weiteren wird zwischen homogenen und heterogenen Ketten unterschieden. Homogene Ub-Ketten bestehen aus Ub-Mole- unterschiedlicher Ub-Ketten modifiziert wer-

külen, die immer durch den gleichen Lysinrest verknüpft sind, während heterogene Ketten über verschiedene Lysinreste verknüpft sind. Unterschiedliche Ketten üben verschiedene Funktionen in der Zelle aus. K48-verknüpfte Ketten sind z. B. das vorwiegende Signal für den proteasomalen Abbau, während K63-verknüpfte Ketten in nichtproteolytische Funktionen wie DNA-Reparatur und dem NF-KB-Signaltransduktionsweg involviert sind [3].

Für die restlichen fünf homogen verknüpften Ub-Ketten sind nur bedingt Informationen über die jeweilige Funktion verfügbar [4, 5]. K6-verknüpfte Ketten spielen eine wichtige Rolle für DNA-Reparatur sowie Parkin-gesteuerte Mitophagie. K11-verknüpfte Ketten dienen neben K48-verknüpften Ketten als ein wichtiges proteasomales Abbausignal, insbesondere bei der Regulation des Zellzyklus. K27-verknüpfte Ketten hingegen wurden Funktionen bei DNA-Repa-

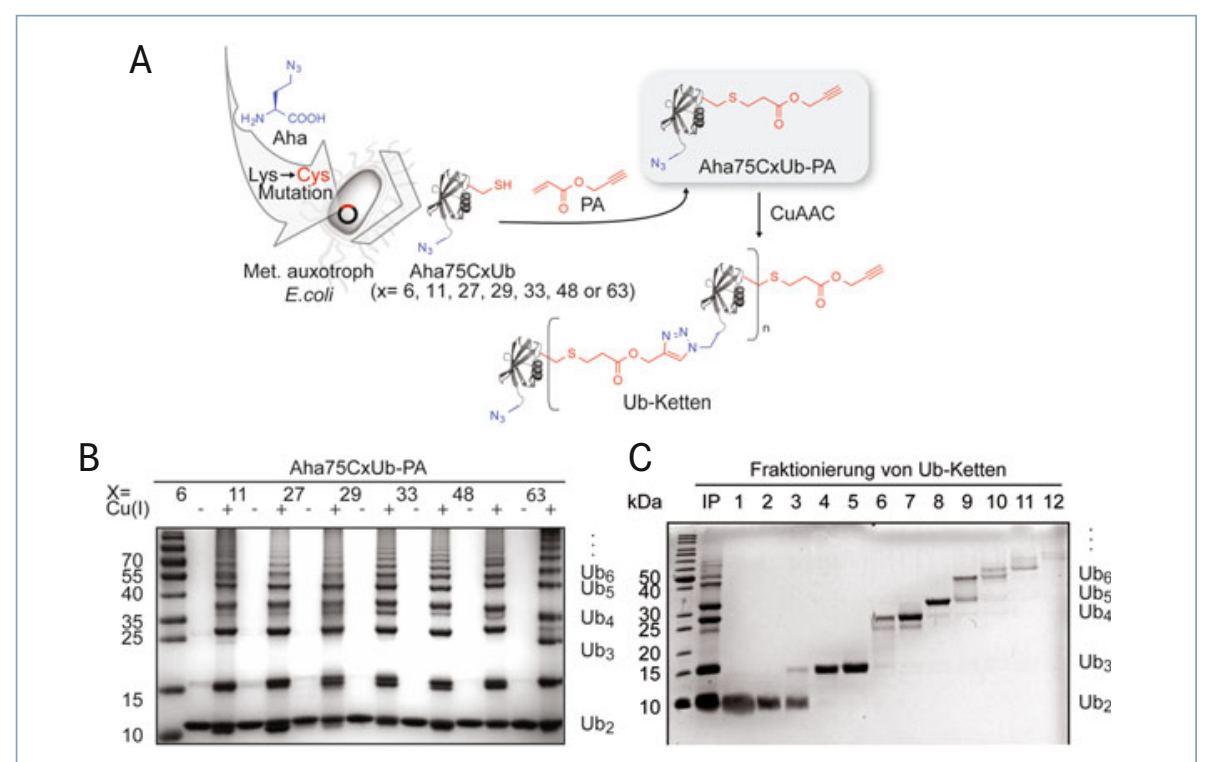

$\Delta$ Abb. 1: Synthese homogener Ubiquitin(Ub)-Ketten. A, Schema zur Herstellung Triazol-verknüpfter Ub-Ketten, einschließlich des Einbaus von Azidohomoalanin (Aha) in Ub in Escherichia coli, bioorthogonaler Modifikation von Ub mittels Propargylacrylat (PA) und Protein-Polymerisierung über CuAAC (copper(I)-catalyzed alkyne-azide cycloaddition). B, Die resultierenden Ketten wurden durch SDS-PAGE aufgetrennt und durch Coomassie-Blue-Färbung gefärbt. C, SDS-PAGEAnalyse der Trennung der Ub-Polymere über GELFrEE-Fraktionierung (gel eluted liquid fraction entrapment electrophoresis): IP Input, 1-12 Elutionsfraktionen mit den jeweiligen Kettenlängen. 
A

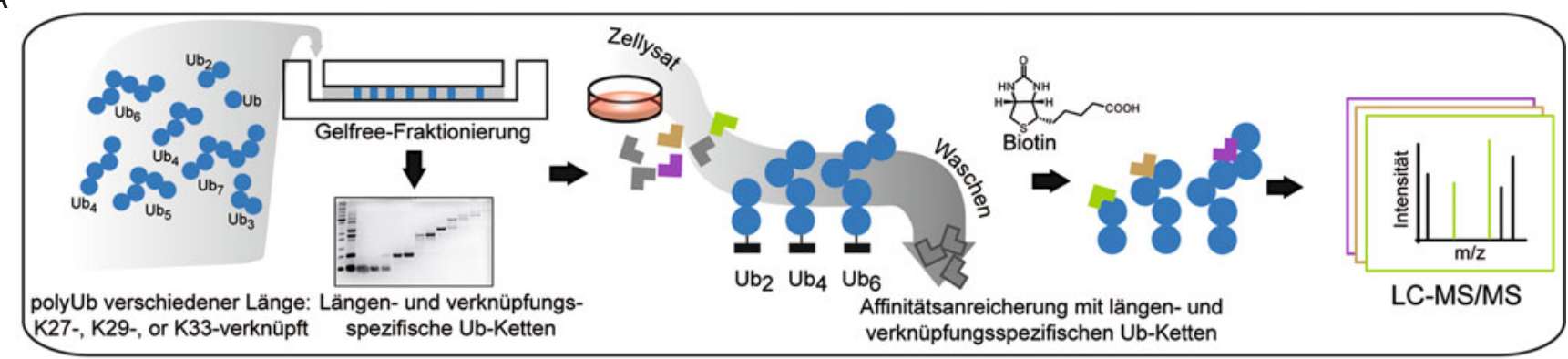

B

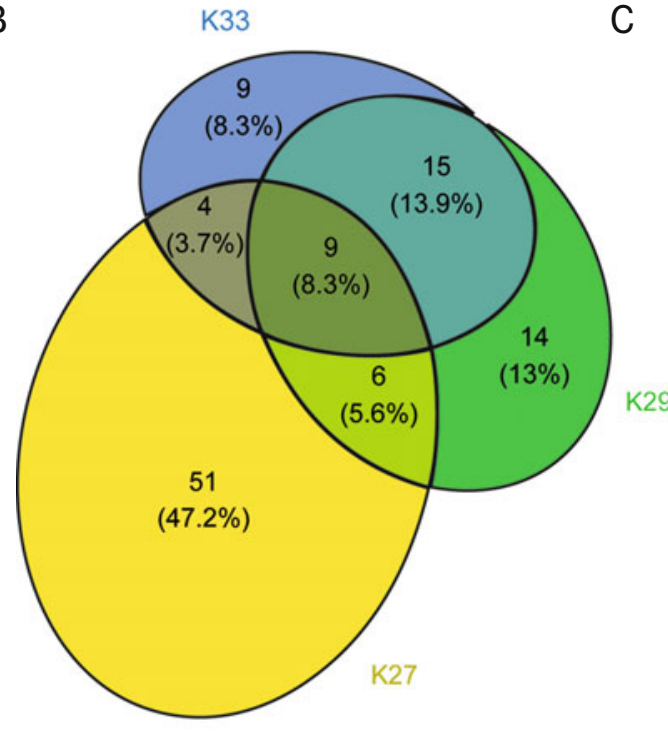

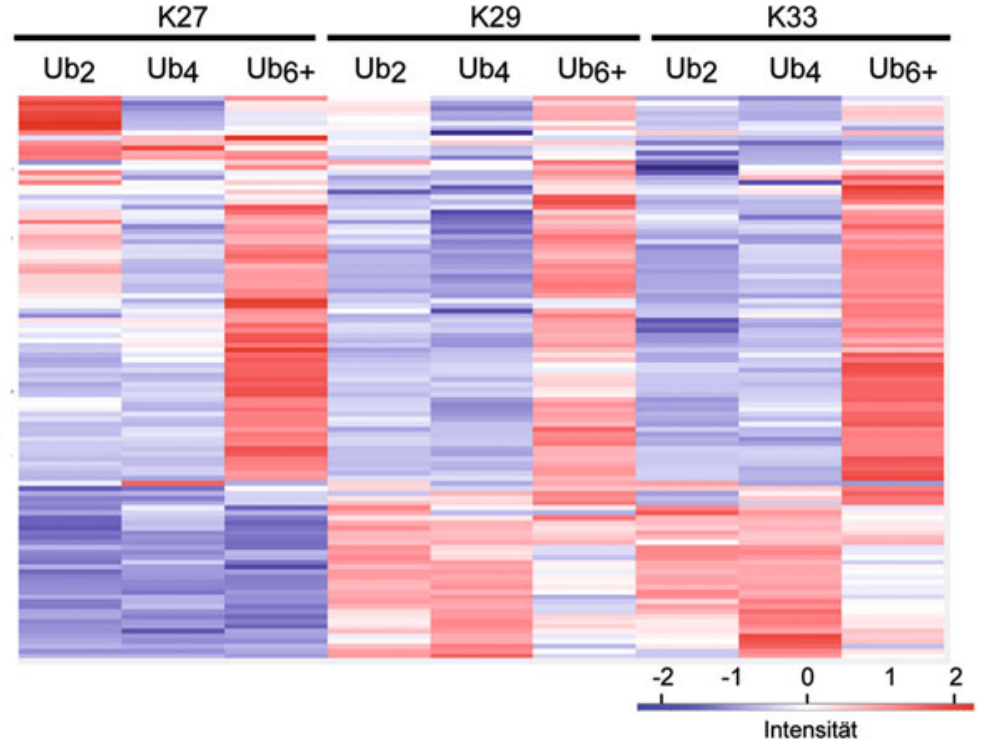

D

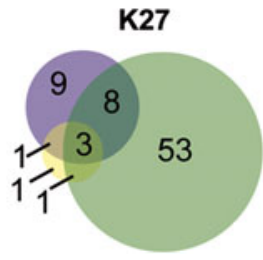

K29

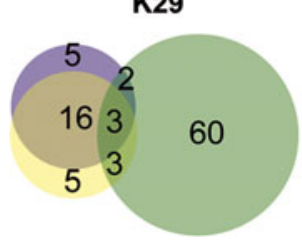

K33

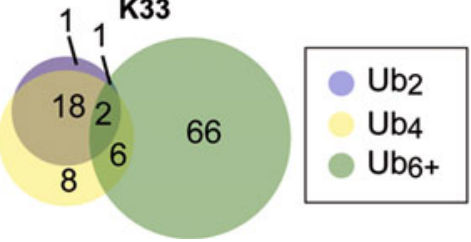

$\Delta$ Abb. 2: Überblick über den Arbeitsablauf für die AP-MS(affinity purification-mass spectrometry)-basierte Identifizierung von Interaktionspartnern von längen- und verknüpfungsspezifischen Ubiquitin(Ub)-Ketten. A, Schema für die GELFrEE-Fraktionierung von verknüpfungsspezifischen Ub-Ketten verschiedener Polymerisierungsgrade. Ub2, Ub4, Ub6+ verknüpft über K27, K29 oder K33 wurden als Affinitätsmatrix für eine Affinitätsanreicherung von UBPs in HEK293T-Zellysat verwendet. Angereicherte Proteine wurden mittels MS-basierter Proteomik identifiziert. B, Überblick über die gemeinsamen und selektiven Interaktionspartner, die anhand der drei Kettentypen (K27, K29, K33) identifiziert wurden. C, Hierarchische Gruppierung von statistisch signifikanten Interaktionen mit längen- und verknüpfungsspezifischen Ub-Ketten. Die interagierenden Proteine sind in den Reihen gezeigt; Spalten zeigen die Ub-Variante die als Affinitätsmatrix benutzt wurde. D, Überblick über längenspezifische Interaktionen. Die Zahlen geben die Anzahl an identifizierten Proteinen an. Nach [11, 12], (C) John Wiley \& Sons, Inc.

ratur, Proteinsekretion und Autophagie zugeordnet. K33-Ketten wurden mit verschiedenen Prozessen wie TCR-Signaltransduktion und post-Golgi-Membrantransport in Verbindung gebracht. Zusammen mit K29-verknüpften Ketten sind K33-verknüpfte Ketten an der Regulation von AMPK(AMP-aktivierter Proteinkinase)-ähnlichen Proteinkinasen beteiligt. Weiterhin dienen K29-verknüpfte Ketten wahrscheinlich als Signal für den Abbau durch das Proteasom.

\section{Signaltransduktion durch Ubiquitin}

Die Vielfalt der möglichen Ubiquitinmodifikationen erlaubt es daher, für eine große Anzahl unterschiedlicher zellulärer Signale zu codieren (Ub-Code). Allgemein wird angenommen, dass die Topologie der jeweiligen Ubiquitinkette das Schicksal des modifizierten Substratproteins beeinflusst. Dabei spielt vor allem die Position der Verknüpfung innerhalb der Kette und die daraus resultierende räumliche Anordnung und
Konformation der Ub-Moleküle zueinander eine wichtige Rolle [6], da diese von spezifischen Ub-Bindeproteinen (UBP) erkannt wird. Ein generelles Modell, wie UBP spezifische Charakteristika wie Topologie und Konformation von Ub-Ketten selektiv erkennen, besteht bisher jedoch nicht. Strukturelle Studien mit Komplexen von UBP und UbKetten deuten deuten jedoch darauf hin, dass die charakteristische Anordnung von Ub-bindenden Domänen (UBD) in den UBP 
zur spezifischen Erkennung von Ub-Ketten maßgeblich beitragen.

Neben der Art der Verknüpfung könnte auch die Kettenlänge ein weiteres, noch weniger verstandenes Charakteristikum für die Erkennung von Ub-Ketten darstellen. Einzelne Beispiele für die potenzielle Bedeutung der Ub-Kettenlänge sind bereits bekannt. So wurde z. B. gezeigt, dass das deubiquitylierende Enzym (DUB) USP5 UbTetramere mit deutlich höherer Affinität bindet als Dimere und dass das DUB UCH-L3 bevorzugt kurze Polymere schneidet [7]. Als weiteres Beispiel dient das proteasomale Transportprotein hRad23A, das präferiert lange K48-verknüpfte Ub-Ketten bindet [8]. Diese Beispiele geben einen ersten Hinweis darauf, dass die Länge einer Ub-Kette neben dem Verknüpfungstyp ein weiteres wichtiges Element des Ub-Codes darstellt.

Allerdings gab es bis vor kurzem keine Studie, die den Einfluss der Kettenlänge auf die Erkennung von Ub-Ketten generell zum Forschungsgegenstand hatte.
Herstellung definierter Ubiquitinketten Für Studien von spezifisch verknüpften UbKetten ist vor allem die Herstellung letzterer in ausreichender Menge und Reinheit essenziell. Enzymatische und synthetische Methoden, die Festphasensynthese sowie chemische Prozessierung von Ub-Molekülen beinhalten, wurden entwickelt, um diese Hürden zu überwinden [9]. Beide Ansätze weisen jedoch auch Nachteile auf, wie hoher Arbeitsaufwand, geringe Kettenlänge und Unspezifität der verwendeten Enzyme. Ein weiterer, wesentlicher Nachteil der so generierten „nativen“ Ub-Ketten ist ihre Anfälligkeit für hydrolytische Spaltung durch DUB. Dies führt zu einer deutlichen Beschränkung ihrer Anwendungsmöglichkeiten in Zellextrakten. Um dieser Einschränkung zu begegnen, haben wir einen Ansatz entwickelt, der den Einbau einer einzelnen unnatürlichen Aminosäure in Ub über die selective pressure incorporation-Methode (SPI) [10] mit Michael-Addition sowie mit der Kupfer(I)katalysierten Azid-Alkin-Zykloaddition (CuAAC) kombiniert (Abb. 1, [11]).

\section{Identifizierung von verknüpfungs- und längenspezifischen UBP}

Die mithilfe unseres Ansatzes erzeugten DUB-resistenten Polymere haben wir in HEK293T-Zellysat eingesetzt, um dort mittels eines auf Affinitätschromatographie und Massenspektrometrie basierenden Ansatzes ketten- und längenspezifische UBP zu identifizieren (Abb. 2A). Dazu wurden die jeweiligen Ub-Ketten mit einem DesthiobiotinAffinitätstag versehen, wodurch sie auf Streptavidin-Agarose immobilisiert werden konnten. Entscheidend hierfür war, dass wir zusätzlich zur Darstellung von Ub-Ketten eines definierten Verknüpfungstyps ein Verfahren für die längenspezifische Trennung der durch CuAAC erzeugten verschiedenen Polymerisierungsgrade, entwickelt haben [12]. Durch gelbasierte FlüssigfraktionGelektrophorese (GELFrEE-Fraktionierung) gelang eine Trennung der Ub-Polymere bis auf Tetramerebene. Längere Ketten wurden als Mischung erhalten (Abb. 1C). Durch die anschließende Verwendung der definierten Ub-Ketten als Affinitätsmatrix konnten wir

\section{Hier steht eine Anzeige.}

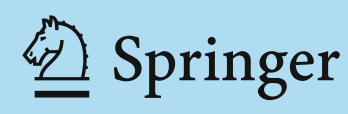


aus HEK293T-Zellysat zahlreiche Proteine anreichern und identifizieren, die spezifisch mit den wenig charakterisierten K27-, K29-, und/oder K33-verknüpften Ketten interagieren (Abb. 2B). Des Weiteren zeigen unsere Affinitätsstudien mit längendefinierten UbKetten $\left(\mathrm{Ub}_{2}, \mathrm{Ub}_{4}, \mathrm{Ub}_{6+}\right)$, dass neben dem Verknüpfungstyp auch die Kettenlänge ein wichtiges Element für die spezifische Erkennung von Ub-Ketten darstellt (Abb. 2C, D). Besonders auffallend ist, dass eine große Zahl von UBP präferiert mit längeren Ub-Ketten eines Verknüpfungstyps interagiert.

Bisherige Studien zeigen, dass Ub-Ketten eines bestimmten Verknüpfungstyps nicht nur eine einzige Konformation, sondern eher ein für diesen Verknüpfungstyp spezifisches Ensemble von Konformationen annehmen [13]. In diesem Zusammenhang erscheint es wahrscheinlich, dass lange Polymere eine größere Anzahl verschiedener Konformationen annehmen können als z. B. Dimere und dadurch mehr Möglichkeiten für spezifische Interaktionsstellen gegeben sind. Dies würde längeren Ketten erlauben, mehr Orientierungen von spezifischen Interaktionsstellen zur Verfügung zu stellen als kurze Ketten. Unsere Studie liefert somit klare Anhaltspunkte, dass die Kettenlänge einen wichtigen Einfluss auf die Erkennung verschiedener UbKetten durch UBP hat und dadurch auch unmittelbar das Schicksal des entsprechend modifizierten Proteins beeinflusst.

\section{Ausblick}

Der hier vorgestellte Ansatz birgt großes Potenzial für die Untersuchung von bisher wenig verstandenen Ub-Modifizierungen. Dazu gehören z. B. heterogene Ub-Ketten, die verschiedene Verknüpfungspunkte aufweisen oder auch verzweigte Ketten. Weiterhin kann der Effekt von Modifizierungen von Ubiquitin selbst, durch z. B. Phosphorylierung, Acetylierung oder SUMOylierung, auf die Erkennung durch UBP untersucht werden. Ebenfalls von großem Interesse ist es, über strukturelle Studien die genauen molekularen Mechanismen aufzuklären, über die die Kettenlänge von spezifischen UBP erkannt wird. Der hier vorgestellte Ansatz stellt somit ein überaus vielfältiges Werkzeug dar, um die biochemischen und zellulären Funktionen der großen Anzahl verschiedenartiger Ubiquitinmodifikationen untersuchen und besser verstehen zu können.

\section{Literatur}

[1] Komander D, Rape M (2012) The ubiquitin code. Annu Rev Biochem 81:203-229

[2] Popovic D, Vucic D, Dikic I (2014) Ubiquitination in disease pathogenesis and treatment. Nat Med 20:1242

[3] Scheffner M, Nuber U, Huibregtse JM (1995) Protein ubiquitination involving an E1-E2-E3 enzyme ubiquitinnthioester cascade. Nature 373:81-83

[4] Kulathu Y, Komander D (2012) Atypical ubiquitylation the unexplored world of polyubiquitin beyond Lys48 and Lys63 linkages. Nat Rev Mol Cell Biol 13:508-523

[5] Swatek KN, Komander D (2016) Ubiquitin modifications. Cell Res 26:399-422

[6] Alfano C, Faggiano S, Pastore A (2016) The ball and chain of polyubiquitin structures. Trends Biochem Sci 41:371-385

[7] Fushman D, Wilkinson KD (2011) Structure and recognition of polyubiquitin chains of different lengths and linkage. F1000 Biol Rep 3:1-10

[8] Raasi S, Orlov I, Fleming KG, Pickart CM (2004) Binding of polyubiquitin chains to ubiquitin-associated (UBA) domains of HHR23A. J Mol Biol 341:1367-1379

[9] Spasser L, Brik A (2012) Chemistry and biology of the ubiquitin signal. Angew Chem Int Ed 51:6840-6862

10 Rosner D, Schneider T, Schneider D et al. (2015) Click

chemistry for targeted protein ubiquitylation and ubiquitin chain formation. Nat Protocols 10:1594-1611

[11] Zhao X, Lutz J, Höllmüller E et al. (2017) Identification of proteins interacting with ubiquitin chains. Angew Chem Int Ed 56:15764-15768
[12] Lutz J, Höllmüller E, Scheffner M et al. (2020) The length of a ubiquitin chain is a general determinant for selective recognition by ubiquitin-binding proteins. Angew Chem Int Ed 59: 12371-12375

[13] Castañeda CA, Chaturvedi A, Camara CM et al. (2016) Linkage-specific conformational ensembles of non-canonical polyubiquitin chains. Phys Chem Chem Phys 18:5771-5788

\section{Funding Open Access funding provided by Projekt DEAL.}

Open Access Dieser Artikel wird unter der Creative Commons Namensnennung 4.0 International Lizenz veröffentlicht, welche die Nutzung, Vervielfältigung, Bearbeitung, Verbreitung und Wiedergabe in jeglichem Medium und For ordnungsgemä $B$ nennen, einen Link zur Creative Commons Luelle ordnungsgemäß nennen, einen Link zur Creative Commons Lizenz beifü
angeben, ob Änderungen vorgenommen wurden. Die in diesem Artikel angeben, ob Anderungen vorgenommin witen. Die in diesem Artikel
enthaltenen Bilder und sonstiges Drittmaterial unterliegen ebenfalls der genannten Creative Commons Lizenz, sofern sich aus der Abbildungslegende nichts anderes ergibt. Sofern das betreffende Material nicht unter der genannten Creative Commons Lizenz steht und die betreffende Handlung nicht nach gesetzlichen Vorschriften erlaubt ist, ist für die oben aufgeführten

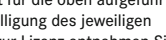
Rechteinhabers einzuholen. Weitere Details zur Lizenz entnehmen Sie bitte der Lizenzinformation auf http///creativecommons.org/licenses/by/4.0/deed.de.

Korrespondenzadresse:

Prof. Dr. Florian Stengel

Fachbereich Biologie

Konstanz Research School Chemical Biology

Universität Konstanz

Universitätsstraße 10

D-78457 Konstanz

florian.stengel@uni-konstanz.de

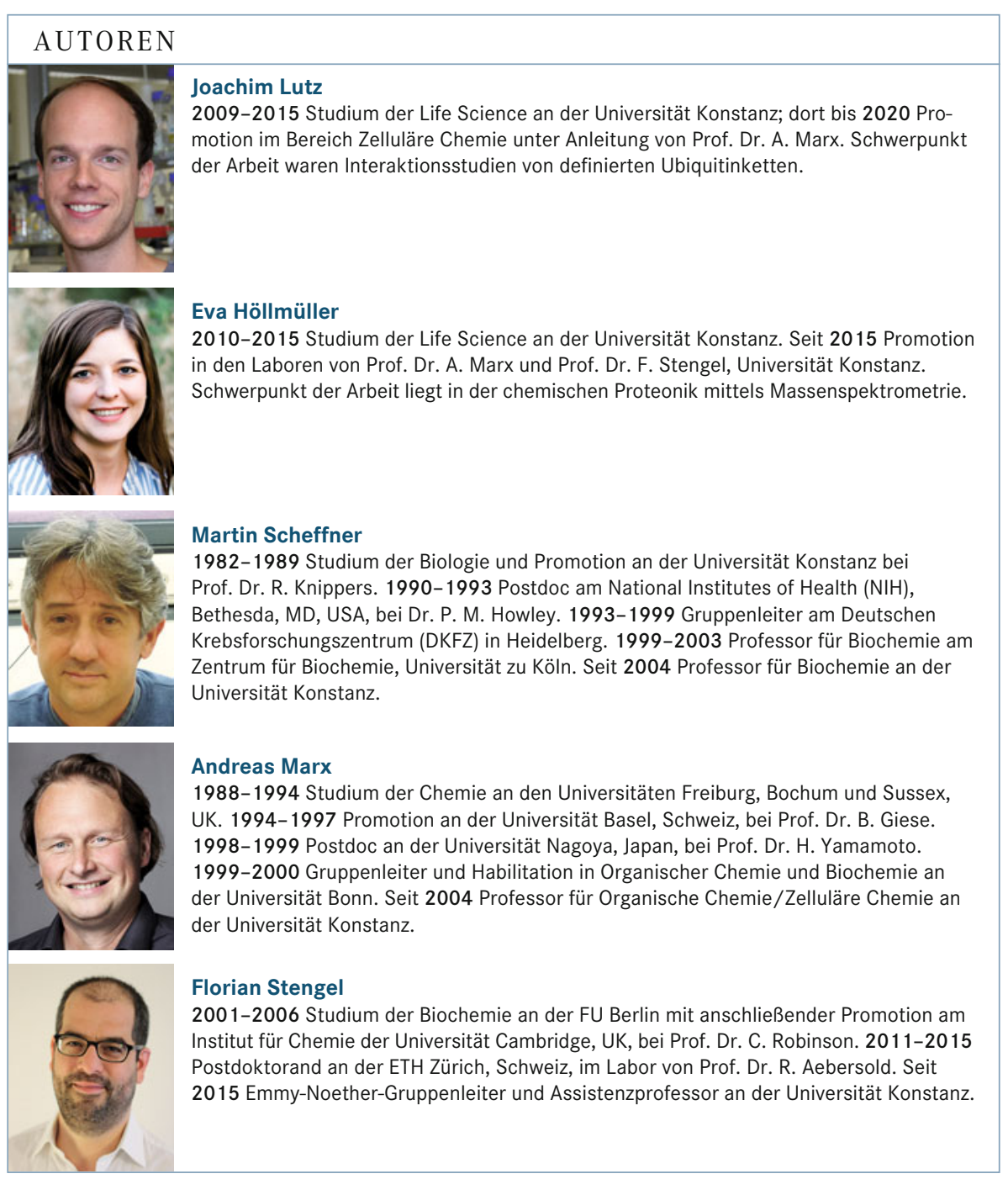

